Research Article

\title{
Scheduling Just-in-Time Transport Vehicles to Feed Parts for Mixed Model Assembly Lines
}

\author{
Yunfang Peng $(\mathbb{D}$, Tian Zeng, Yajuan Han $(\mathbb{D}$, and Beixin Xia $\mathbb{D}$ \\ School of Management, Shanghai University, Shanghai 200444, China \\ Correspondence should be addressed to Beixin Xia; bxxia@shu.edu.cn
}

Received 1 November 2019; Revised 11 March 2020; Accepted 20 March 2020; Published 27 April 2020

Academic Editor: Manuel De la Sen

Copyright (c) 2020 Yunfang Peng et al. This is an open access article distributed under the Creative Commons Attribution License, which permits unrestricted use, distribution, and reproduction in any medium, provided the original work is properly cited.

In order to solve the problem of vehicle scheduling to feed parts at automobile assembly line, this study proposes a just-in-time delivery method combined with the mode of material supermarket. A mixed integer linear programming model with the primary objective of using the least number of tow trains is constructed by considering capacity of vehicle and inventory levels of line. On the basis of the minimum number of tow trains, the schedule of each tour is reasonably planned to minimize inventory of assembly line, which is the secondary objective of the part supply problem. Additionally, a heuristic algorithm which can obtain a satisfactory solution in a short time is designed to solve large-scale problems after considering continuity and complexity of modern automobile production. Furthermore, some cases are analyzed and compared with the widely used periodic delivery strategy, and the feasibility of just-in-time model and algorithm is verified. The results reveal that just-in-time delivery strategy has more advantages in reducing inventory level than periodic delivery strategy.

\section{Introduction}

With the increasing demand for multiple varieties and customization, products incorporating customer's individual demands have gradually become mainstream products in the market, making competition in manufacturing industry more and more intensified. Manufacturing industry is the mainstay of national economy. The introduction of Germany's "Industry 4.0" and "Made in China 2025" triggered a new wave of manufacturing revolution. Under the influence of intelligent manufacturing, enterprises desire for new development and occupy a dominant position in increasingly fierce competition. They need to continuously meet the individual needs of customers, enrich product variety, reduce manufacturing cost, and improve labor efficiency and products quality with a shorter production time. It is important to enhance the core competitiveness through production management optimization.

In recent years, the automobile industry, which is mainly engaged in assembly production, has changed its manufacturing mode from few varieties and large quantities to many varieties and small quantities. More and more manufacturers prefer to use mixed model assembly lines instead of rigid production lines for a single product, allowing different models to be assembled on a same production line. Mixed model assembly line has the ability to manufacture multivariety mixed products with similar structure and function, which can improve production efficiency. For example, at the automobile plant of German car producer BMW, more than 13,000 containers delivered by about 600 suppliers on more than 400 trucks need to be processed each day [1]. Production logistics has become one of the major challenges in automobile production. As a result, a reliable and flexible part feeding system is indispensable.

In the automotive industry, part supply accounts for $20 \%-50 \%$ of production costs [2]. If some important parts are not delivered in time, manufacturers will face a risk of stopping the line, causing huge losses. And space between stations is extremely narrow and cannot contain a large amount of inventory. Therefore, an efficient parts supply system needs to deliver required parts (type and quantity) to right place (consumed station) in right way at right time. 
In order to meet part requirements of mixed model assembly line, frequent deliveries with small batch are becoming a trend recently. A new material supermarket was introduced to promote just-in-time part supply for mixed model assembly line in some manufacturers' production workshops. Material supermarket, instead of traditional central warehouse, is directly located next to production line for stacking parts. In a material supermarket, parts are sorted and stored on the shelves for easy access. Material supermarket ensures the implementation of just-in-time part supply strategy, which is thought to be the development of manufacturing industry.

The part feeding problem is an important part of production logistics, which refers to the logistics process of delivering the matched parts to the corresponding stations on time and in accordance with the material requirements and consumption of each station. This study focuses part feeding problem with material supermarket and some tow trains located next to mixed model assembly line, comprehensively considering the capacity of tow train and inventory of assembly stations, and makes decisions on the quantity of tow trains and schedule of each tour and loading parts type, such that the stations never starve for parts while also minimizing line-side inventory. The remainder of this study is structured as follows. In Section 2, an overview of part supply literature is presented. Section 3 describes the optimization problem in detail and gives relevant conditions and assumptions. In Section 4, according to the defined parameters and variables, a mixed integer linear programming model with the objective to minimize the quantity of vehicles and the inventory of assembly stations is established. Considering complexity of actual production, a heuristic algorithm for solving large-scale problem instances is designed in Section 5. In Section 6, the feasibility and effectiveness of the model and algorithm are verified by comparison with traditional periodic delivery. Finally, Section 7 concludes the study and extends new research direction.

\section{Literature Review}

Faced with complex and diverse parts in workshop, many practitioners and scholars have conducted extensive research on their supply. Kilic and Boysen reviewed the problem of assembly line parts supply. Kilic paid attention to three aspects such as parts storage, parts transportation, and feeding policy and focused on objectives and solutions of feeding policy [3]. Boysen et al. detailed the elementary process steps of part logistics in automotive industry and specified important decision-making problems and research challenges in each specific step [4].

Due to the limited space in workshop, many automanufacturers are using material supermarkets in order to achieve small batch supply consistent with just-in-time part supply principle. Battini described the concept of material supermarket in detail and divided it into fishbone supermarket, single-line supermarket, and multiline supermarket. $\mathrm{He}$ also carried out literature research on basic decisionmaking problems that occurred when implementing material supermarkets and solved the location problem of supermarket in production environment with multiple parallel assembly lines $[1,5,6]$.

The part supply process with material supermarket concept amounts to a complicated assignment where several related decisive problems have to be solved:

(i) Number and location of material supermarkets and the assembly line supplied by each supermarket

(ii) Quantity of tow trains per material supermarket and the route of each tow train

(iii) The schedule of each tow train to supply parts on its route

(iv) The bins to be loaded in each tour of a tow train

In spite of material supermarket and tow train widespread application in the automobile, there are only a few literatures definitely dealing with the above problems. Emde and Boysen consider the problem: (i) they discuss the optimal number and placement of supermarkets on the shop floor and present a mathematical model to be solved by using the dynamic programming algorithm [7]. Emde et al. researched the loading problem (iv), which aims at minimizing inventory while avoiding part shortages given the limited capacity of tow trains [8].

Diversification of demand exacerbates complexity and uncertainty of manufacturing environment. Facing a large variety of parts, whether they can be delivered on time will directly affect smooth of production. Therefore, it is necessary to design a part supply route, optimize the vehicle quantity (ii), and make schedule for each tour (iii).

Vaidyanathan first proposed a mathematical model of just-in-time distribution of vehicle route (ii) and assigned the same route to vehicle by assuming a constant demand rate so that a vehicle continuously and repeatedly served during planning horizon [9]. Choi and Lee estimated the consumption rate of parts and analyzed distribution sequence of various parts to form a distribution route, with an improved vehicle route method [10]. Emde comprehensively considered the routing and scheduling problems of vehicles, decides partition, and seeks the optimal route through reasonable division of distribution vehicles and supply stations [11]. Rao et al. considered a single vehicle and decided the target station and driving route of a delivery vehicle according to assembly sequence so that the total delivery and inventory costs are minimized [12]. Different from vehicle routing problem, the optimization of part supply route in assembly workshop is not simply to visit each demand point. It not only needs to meet just-in-time part supply requirements but also should control production cost reasonably. In terms of the quantity of delivery vehicles, based on assumptions and constraints in real cases, Fathi proposed an integer programming model, which aims to use the minimum quantity of vehicles to transport raw materials on assembly line [13]. Golz et al. developed a twostage heuristic decomposition procedure to select paths from predefined sets and minimize the number of drivers; however, it did not establish a normative mathematical model [14].

The scheduling problem of tow trains (iii) in most studies has focused on establishing an appropriate delivery time window or directly using a periodic delivery method. 
Muller studied the vehicle scheduling problem with soft time windows, considered penalty function that violates time window, and used a two-stage heuristic algorithm to calculate approximate solution of the Pareto optimal solution [15]. Zhong et al. considered the problem of rolling stock scheduling with maintenance requirements, and the optimized schedules lead to a reduction in operating cost [16]. The periodic delivery method means that a vehicle is transported at the same time interval. Schneider and Emde first minimize the quantity of vehicles and then minimize total route duration so that vehicles with capacity can periodically execute their assigned routes, but the quantity of parts loaded is not clearly resolved [17]. Zhang et al. introduced an extended time-space network construct for the cyclic train timetabling problem [18]. Kilic et al. put forward that it is important to send materials to stations periodically. With a purpose of reducing work-in-process and transportation costs, they developed a periodic material transportation model for vehicle operation [19]. Fathi et al. assumed that material handling occurred periodically at the same time interval, by introducing heuristic algorithms to find the optimal loading and parts supply sequence for a vehicle [20]. Emde and Gendreau analyzed the cyclic distribution of vehicles and solved the problem of scheduling and loading of vehicles with predefined routes [21]. Fathi solved the scheduling problem more directly, allowing transportation tours to be deleted in a fixed schedule, minimizing the number of tours and inventory levels [22]. Periodic delivery, using same time interval, simplifies the vehicle scheduling problem, which makes vehicle less flexible and unable to obtain a lower inventory. A summary of relevant literature is shown in Table 1.

At present, the competition situation of automobile manufacturing industry is becoming increasingly fierce. The key for enterprises to reduce production cost is to establish efficient material distribution system, reduce unnecessary waste in the production process, and optimize and improve the production logistics process. Boysen et al. decided on the number of logistics workers for part feeding to minimize distribution costs [23]. Emde and Gendreau tackled the part supply problem so that the stations never lack parts while minimizing line-side inventory [21]. Accorsi et al. provided a decision-support system for part supply to achieve total travel distance and save costs [24]. Zhou and Shen considered energy consumption and proposed an energy-efficient scheduling method to reduce material distribution costs [25]. And Qiu et al. also investigate the energy consumption of AGVs, finding an optimization route to decrease the energy consumption [26].

Although previous studies focused on the part supply problem, material supermarket and tow train have been used for part supply. This study differs from existing literature in three ways. First, the part supply model with material supermarket in this article considers the quantity of vehicles (ii), schedule of each tour (iii), and type of parts loaded per tour (iv) simultaneously. But most of researches solved only one or two of the four related decision problems. Second, when involving schedule of tours, most studies are devoted to use a periodic delivery method. This study finds out a just- in-time part supply method which could make the schedule of each tour with the shortest parts storage time. As a result, the inventory cost and operating cost are resonably reduced. Third, according to the characteristics of part feeding problems, we design a heuristic algorithm for solving largescale problems, which can obtain satisfactory solutions to problems in a short time.

\section{Problem Description}

Material supermarkets are decentralized logistics areas which factory used for storing raw material, finished product, and semimanufactured products. Many manufacturers begin to use material supermarket for frequent small batch supply in order to realize just-in-time part supply principle. Parts from suppliers are transported to workshop by industrial trucks and then stored in material supermarket. The delivery of parts to production line is carried out by small tow trains. The layout of an assembly line is shown in Figure 1. Material supermarket is located near assembly line, containing a number of tow trains that supply parts for stations of assembly line. A tow train is loaded in material supermarket with bins of parts; then, it sets off to visit some stations that lie on its predefined route. When tow train arrived at the storage location of each part, it will unload part bins and recycle empty bins. Once it has accomplished this tour, it will return to material supermarket. When a new delivery tour is generated, tow train performs picking operation, and it will be waiting until the best time to transport part bins to stations. In this material supermarket with tow trains distribution system, part requirements can be obtained through product sequence and make decisions on the number of tow trains, schedule of each tour, and loading parts type; therefore, the stations never starve for parts while minimizing line-side inventory.

According to literature of part supply [11], to precisely describe the part feeding problem, some assumptions are introduced as follows:

(1) All tow trains parked in the material supermarket are perfect and trouble-free equipment. Tow train travels at a uniform speed on its route, and travel time is constant, regardless of the acceleration and deceleration processes.

(2) Parts are packaged in standard bins. Tow train loads part bins in material supermarket and unloads part bins at station. It requires a certain loading and unloading time. Loading and unloading times for one bin of different parts are the same.

(3) Once departed from material supermarket, a tow train will always cycle through all stations on its route without interruption. It is not permitted for a tow train to give up some or all tasks in process of transportation.

(4) Capacity of tow train is limited. The number of part bins loaded each tour cannot exceed its capacity. For each part, only one bin can be loaded in each tour. In addition, repeated loading is not allowed. 
TABLE 1: Summary of related literature.

\begin{tabular}{|c|c|c|c|c|c|c|c|c|c|}
\hline \multirow{2}{*}{ Researchers } & \multicolumn{4}{|c|}{ Decision problem } & \multicolumn{2}{|c|}{$\begin{array}{l}\text { Delivery } \\
\text { strategy }\end{array}$} & \multicolumn{3}{|c|}{ Methodology } \\
\hline & $\begin{array}{c}\text { Number and location } \\
\text { of supermarket }\end{array}$ & $\begin{array}{c}\text { Number and route } \\
\text { of tow trains }\end{array}$ & Scheduling & Loading & JIT & Periodic & Exact & Heuristic & Metaheuristic \\
\hline $\begin{array}{l}\text { Emde and Boysen } \\
\text { [7] }\end{array}$ & $\checkmark$ & & & & $\checkmark$ & & $\checkmark$ & & \\
\hline Emde et al. [8] & & & & $\checkmark$ & $\checkmark$ & & $\checkmark$ & & \\
\hline Vaidyanathan [9] & & $\checkmark$ & & & $\checkmark$ & & & $\checkmark$ & \\
\hline Choi and Lee [10] & & $\checkmark$ & & & & & & $\checkmark$ & \\
\hline Emde [11] & & $\checkmark$ & $\checkmark$ & & $\checkmark$ & & $\checkmark$ & & \\
\hline Rao et al. [12] & & $\checkmark$ & & & $\checkmark$ & & & & GA and SA \\
\hline Fathi [13] & & $\checkmark$ & & & & & $\checkmark$ & & \\
\hline Golz et al. [14] & & $\checkmark$ & & & & $\checkmark$ & & $\checkmark$ & \\
\hline Muller [15] & & & $\checkmark$ & & & & & $\checkmark$ & \\
\hline $\begin{array}{l}\text { Schneider and } \\
\text { Emde [17] }\end{array}$ & & $\checkmark$ & & & & $\checkmark$ & & & LNS \\
\hline Zhang et al. [18] & & & $\checkmark$ & & & $\checkmark$ & & $\checkmark$ & \\
\hline Kilic et al. [19] & & & $\checkmark$ & & & $\checkmark$ & & $\checkmark$ & \\
\hline Fathi et al. [20] & & & $\checkmark$ & $\checkmark$ & & $\checkmark$ & & & PSO \\
\hline $\begin{array}{l}\text { Emde and } \\
\text { Gendreau [21] }\end{array}$ & & & $\checkmark$ & $\checkmark$ & $\checkmark$ & & & $\checkmark$ & \\
\hline
\end{tabular}

JIT, just-in-time; GA, genetic algorithm; SA, simulated annealing; LNS, large neighborhood search; PSO, particle swarm optimization.

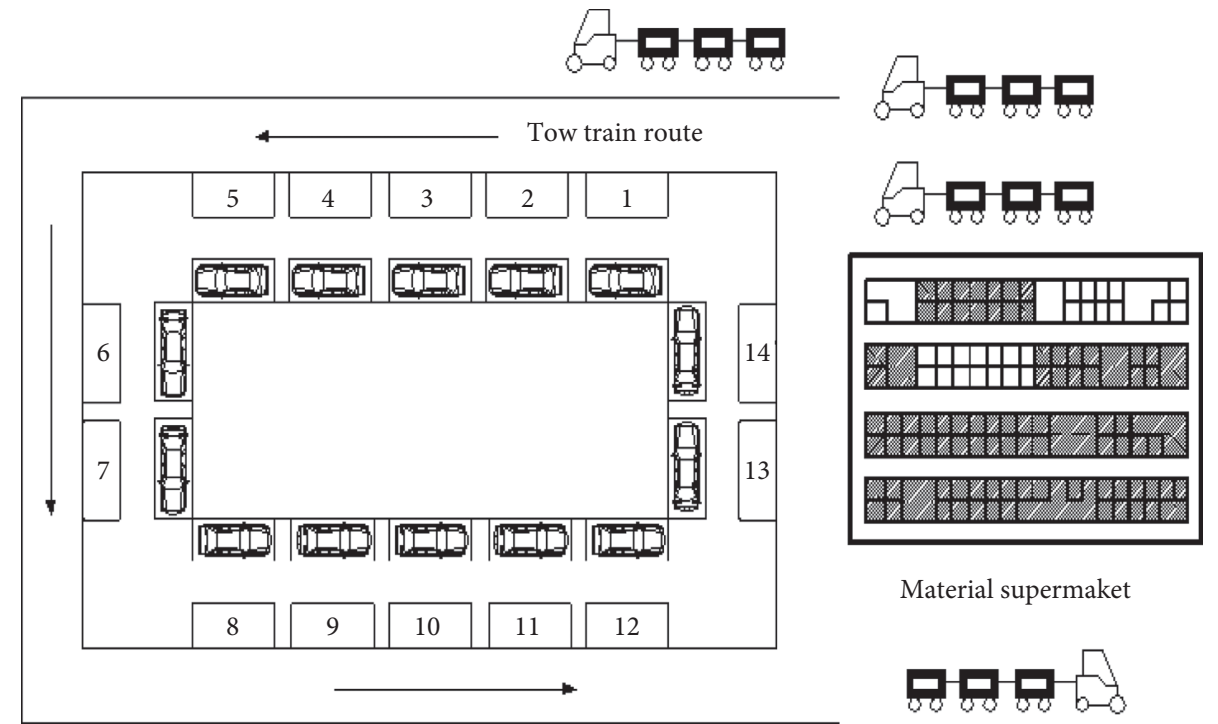

FIGURE 1: Layout of part supply with material supermarket.

(5) To avoid congestions in the narrow aisles, stations on a tow train's route are always consecutive and served in the flow direction of the assembly line.

According to customer's personalized order, the assembly sequence for a given assembly planning period can be known in advance. Models enter stations where some assembly workers assemble them and consume corresponding parts. The type and quantity of parts consumed for each model is obtained from bill of materials (BOMs). Therefore, according to assembly sequence of this planning period, the total parts requirements of each station can be determined. Parts are packaged in standard bins and loaded onto tow trains to transport to stations.
For example, three different models $M_{1}, M_{2}$, and $M_{3}$ need to be assembled on a mixed model assembly line. Five different parts $P_{1}, P_{2}, P_{3}, P_{4}$, and $P_{5}$ are consumed in stations. BOM is shown in Table 2. Depending on customer orders, the total demand for models is to be satisfied by the following given production sequence: $<2,3,1,1,3$, and $2>$. Part requirements of each cycle during the assembly planning period can be calculated, which are shown in Table 3. Assuming that bin capacities of each part is $4,3,5,3$, and 5 , respectively, it can be calculated that bin demand of $P_{1}$ and $P_{2}$ is 2 and bin demand of $P_{3}$, $P_{4}$, and $P_{5}$ is 1 . Bin demand for each cycles can be found in Table 4. According to required time of each part, it is necessary to arrange vehicles so that time for each part to 
TABLE 2: Bill of material (BOM).

\begin{tabular}{cccccc}
\hline & $P_{1}$ & $P_{2}$ & $P_{3}$ & $P_{4}$ & $P_{5}$ \\
\hline$M_{1}$ & 1 & 1 & 1 & 0 & 1 \\
$M_{2}$ & 1 & 0 & 0 & 1 & 1 \\
$M_{3}$ & 1 & 1 & 1 & 0 & 0 \\
\hline
\end{tabular}

TABle 3: Demand of parts per cycle.

\begin{tabular}{ccccccccccc}
\hline & 1 & 2 & 3 & 4 & 5 & 6 & 7 & 8 & 9 & 10 \\
\hline$P_{1}$ & 1 & 1 & 1 & 1 & 1 & 1 & 0 & 0 & 0 & 0 \\
$P_{2}$ & 0 & 0 & 1 & 1 & 1 & 1 & 0 & 0 & 0 & 0 \\
$P_{3}$ & 0 & 0 & 0 & 1 & 1 & 1 & 1 & 0 & 0 & 0 \\
$P_{4}$ & 0 & 0 & 0 & 1 & 0 & 0 & 0 & 0 & 1 & 0 \\
$P_{5}$ & 0 & 0 & 0 & 0 & 1 & 0 & 1 & 1 & 0 & 1 \\
\hline
\end{tabular}

TABle 4: Demand of bins per cycle.

\begin{tabular}{lllllllllll}
\hline & 1 & 2 & 3 & 4 & 5 & 6 & 7 & 8 & 9 & 10 \\
\hline$P_{1}$ & 1 & 0 & 0 & 0 & 1 & 0 & 0 & 0 & 0 & 0 \\
$P_{2}$ & 0 & 0 & 1 & 0 & 0 & 1 & 0 & 0 & 0 & 0 \\
$P_{3}$ & 0 & 0 & 0 & 1 & 0 & 0 & 0 & 0 & 0 & 0 \\
$P_{4}$ & 0 & 0 & 0 & 1 & 0 & 0 & 0 & 0 & 0 & 0 \\
$P_{5}$ & 0 & 0 & 0 & 0 & 1 & 0 & 0 & 0 & 0 & 0 \\
\hline
\end{tabular}

arrive at stations is as close as possible to the time consumed.

Consequently, during the given assembly planning period, taking into account capacity of vehicle and inventory of station, in order to solve the part feeding problem, this study tackles the following three decision problem simultaneously:

(i) Determine the optimal quantity of vehicles. In practice, every supermarket will have three to five tow trains for part supply. When a tow train is assigned to visit stations, it will generate operating cost. If there are not enough tow trains for part supply, part shortages can lead to line-stoppages. But too many tow trains will increase production costs. Therefore, determining the minimum number of vehicles is essential.

(ii) Decide on the schedule of tow trains to execute each tour. While the optimal number of tow trains is decided, each tow train will carry out some tours. When material shortage takes place, manufacturers will face a risk of stopping the line, causing huge losses and assembly workers being idle. So, each tow train's delivery schedule for supplying parts need to be determined.

(iii) Determine the type of parts loaded per tour. Based on part requirements of stations and consumption time, in order to maintain minimum inventory, it is indispensable to decide bins to be loaded each tour of a tow train.

\section{Mixed Integer Linear Programming Model}

This study tackles part supply problem of mixed model assembly line. Different from a scheduling model that uses fixed time intervals, according to the number of tours, a justin-time vehicle scheduling model is established with goal of minimizing the quantity of vehicles and the inventory of assembly stations. The model ensures that vehicle completes delivery task without shortage of parts, and it makes storage time of each part as short as possible, thus reducing inventory of stations. It is beneficial to reduce production management costs.

According to the total demand of part bins and tow train capacity, the minimum number of tours $N$ can be calculated as

$$
N=\left\lceil\sum_{p=1}^{P}\left(\sum_{m=1}^{M} \frac{B_{p m}-h_{0 p}}{C \cdot Q_{p}}\right)\right\rceil .
$$

To describe the model, the following symbol definitions are given:

(1) Sets and indices

$P$ : set of parts

$N$ : set of tours

$M$ : set of cycles

$K$ : set of vehicles

$p$ : part index, $p \in P$

$n$ : tour index, $n \in N$

$m$ : cycle index, $m \in M$

$k$ : vehicle index, $k \in K$

(2) Parameters

$Q_{p}$ : number of parts in a bin

ct: work cycle of assembly line

$T$ : driving time it takes for a tow train to make a full tour

$l$ : time it takes for a tow train to load a bin in supermarket

$r$ : time it takes for a tow train to unload a bin at station

$B_{p m}:$ demand of part $p$ in cycle $m$

$C$ : maximum capacity (in number of bins) that can be loaded onto a tow train

$t_{p}$ : driving time it takes for a tow train to drive from supermarket to part $p$

$h_{o p}$ : initial inventory for part $p$ at beginning of the first tour

$t_{0}$ : time when material delivery schedule starts

$t_{e}$ : time when material delivery schedule ends

$G$ : big enough integer

$g$ : small enough integer

(3) Decision variables

$y_{k}$ : binary variable: 1 , if tow train $k$ uses; 0 , otherwise $Y_{n k}$ : binary variable: 1 , if tour $n$ is performed by tow train $k$; 0 , otherwise

$t_{n k}$ : start time for tow train $k$ to perform tour $n$ $t_{n p k}^{\prime}$ : delivery time for tow train $k$ to transported part $p$ in tour $n$

$X_{n p}$ : binary variable: 1 , if part $p$ is carried in tour $n$; 0 , otherwise 
$H_{m p}$ : inventory of part $p$ after cycle $m$

$D_{n p}$ : total consumption of part $p$ in tour $n$

$z_{n m p}$ : binary variable: 1 , if cycle $m$ starts assembly when part $p$ is transported in tour $n ; 0$, otherwise $u_{m n p}$ : binary variable: 1 , if tour $n$ transports part $p$ arrived at cycle $m$; 0 , otherwise

$e_{n m p}$ : binary variable: 1 , if tour $n$ transports part $p$ in cycle $m$; 0 , otherwise

(4) MILP model

$$
\begin{aligned}
& \operatorname{Min} Z_{1}=\sum_{k=1}^{K} y_{k}, \\
& \operatorname{Min} Z_{2}=\sum_{m=1}^{M} \sum_{p=1}^{P} H_{m p}, \\
& H_{m p}=h_{0 p}+\sum_{j=1}^{N} e_{n m p} * Q_{p}-\sum_{i=1}^{m} B_{p i}, \\
& \quad \forall n \in N, p \in P, m \in M, \\
& \sum_{P=1}^{P} X_{n p} \leq C, \quad \forall n \in N, \\
& Y_{n k} \leq y_{k}, \quad \forall n \in N, k \in K, \\
& \sum_{n=1}^{N} Y_{n k} \geq y_{k}, \quad \forall k \in K, \\
& \sum_{k=1}^{K} Y_{n k}=1, \quad \forall n \in N,
\end{aligned}
$$

$+l * \sum_{p=1}^{P} X_{n, p}, \quad \forall n, n ı \in N, n ı \geq n, k \in K$,

$t_{n k} \geq t_{0}, \quad \forall n \in N, k \in K$,

$t_{n k} \leq t_{e}, \quad \forall n \in N, k \in K$,

$t_{n k p}^{\prime}=t_{n k}+t_{p}+r * \sum_{q=1}^{p} X_{n q}, \quad \forall n \in N, p \in P, k \in K$,

$$
\begin{aligned}
& D_{n p}= \sum_{m=1}^{M} B_{p m} * z_{n m p}, \quad \forall n \in N, p \in P, \\
& m * c t-t_{n k p} \leq\left(1-z_{n(m+1) p}\right) * G, \\
& \quad \forall n \in N, p \in P, m \in M-1, k \in K,
\end{aligned}
$$

$$
\begin{aligned}
& t_{n k p}^{\prime}-m * c t+g \leq z_{n(m+1) p} * G, \\
& \forall n \in N, p \in P, m \in M-1, k \in K, \\
& c t-t_{n k p}^{\prime} \leq\left(1-z_{n(1) p}\right) * G, \\
& \forall n \in N, p \in P, k \in K, \\
& t_{n k p}^{\prime}-c t+g \leq z_{n(1) p} * G, \\
& \forall n \in N, p \in P, k \in K, \\
& m * c t-t_{n k p}^{\prime}+g \leq u_{n m p} * G, \\
& \forall n \in N, p \in P, m \in M, k \in K \text {, } \\
& t_{n k p}^{\prime}-m * c t \leq\left(1-u_{n m p}\right) * G, \\
& \forall n \in N, p \in P, m \in M, k \in K, \\
& 2 e_{n m p} \leq X_{n p}+u_{n m p}, \quad \forall n \in N, p \in P, m \in M, \\
& 1+e_{n m p} \geq X_{n p}+u_{n m p}, \quad \forall n \in N, p \in P, m \in M, \\
& h_{0 p}+\sum_{j=1}^{n-1} Q_{p} * X_{j p}-D_{n p} \geq 0, \quad \forall n \in N, p \in P, \\
& h_{0 p}+\sum_{n=1}^{N} Q_{p} * X_{n p}-\sum_{m=1}^{M} B_{p m} \geq 0, \quad \forall n \in N, p \in P, m \in M .
\end{aligned}
$$

Objective function (2) shows that the minimum number of tow trains is the primary objective, and objective function (3) shows that the minimization of total inventory at stations is the secondary objective. This is a two-stage progress to solve the model; the first obtain solutions satisfying all constraints that optimize the primary objective. Then, the minimization of secondary objective is restricted on the optimal solution space of the primary objective. Constraint (4) represents inventory of part $p$ after $m$ cycle. Constraint (5) ensures that tow train is not overloaded. Constraint (6) enforces that the number of tow train used per tour does not exceed the maximum value of available vehicles, and (7) states that when a tow train used in part supply, it at least delivery one tour. Constraint (8) ensures that only one tow train can be used for each tour, and constraint (9) defines that when the same tow train is carried out at two different tours, adjacent transport time cannot overlap. Constraints (10) and (11) define the schedule of each tour including start time and end time. Constraint (12) calculates delivery time of tow train $k$ transports part $p$ in tour $n$. Constraints (13)-(17) calculate the total consumption of part $p$ at time of tour $n$. In just-in-time distribution, because the start time of each tour is a decision variable, it is necessary to use binary variable $z_{n m p}$ to determine the range of cycles at which tour $n$ is delivered. Thus, it could obtain total consumption of part $p$ in tour $n$. Constraints (18)-(21) ensure that part $p$ is available at cycle $m$, and $X_{n p}$ and $u_{n m p}$ are used to determine the range of $e_{n m p}$. Constraint (22) ensures that the number of 
all parts after the last tour has been able to maintain consumption before next tour. Constraint (23) allows the total number of transport parts and initial inventory to meet the total number of parts required to assemble product.

\section{Heuristic Algorithm}

With the expansion of part supply problem, it is more difficult to solve the mathematical model. The default solver and exact solution tool can only find the optimal solution for small-scale problems in a limited time. Hence, in order to solve large-sized practical problems, a heuristic algorithm needs to be developed. Based on the optimal number of vehicles, a tow train is arranged to perform a delivery tour. Each tow train's delivery schedule and part bins to be loaded per tour need to be determined.

According to the characteristics of the just-in-time delivery problem, the design of the heuristic algorithm in this paper is reasonable and could obtain satisfactory solutions to the part feeding problem in a short time and shows superior performance. The heuristic algorithm in this paper simulates the production process and consists of four main steps: calculate shortage point, arrange initial tour, tow train allocation, and transport start time coordination. Firstly, input number of cycles, calculate the total demand of parts, and obtain the minimum number of tours, according to production time; hence, it will decide an initial quantity of tow trains and then determine each tow train's initial schedule and part bins to be loaded per tour. Next, each tour will allot to a tow train; if tow train does not have enough time to delivery, the initial schedule of tow train needs to adjust until finding right start time or to increase a new tow train. Finally, on the basis of right schedule and part bins to be loaded, calculate the total inventory. The heuristic algorithm flow chart is shown in Figure 2.

5.1. Calculate Shortage Point. According to the number of cycles, types, and quantities of parts that need to be transported during assembly, a planning period can be obtained. In order to prevent the occurrence of parts shortage, consumption changes in each part should be recorded, and then, the shortage point of each part could be determined.

First step: input number of cycles $(M)$ and determine the type of parts to be transported and the number of bins; it is easy to calculate the total number of bins $(W)$ as follows:

$$
W=\left\lceil\sum_{P=1}^{P}\left(D_{p}-h_{0 p}\right) / Q_{p}\right\rceil .
$$

Second step: according to part type $(P)$, record changes in part consumption. And calculate the time point when each part is used up and the next point in time when a part used is the shortage point $\left(O_{p s}\right)$. For parts requiring multiple bins, it is necessary to calculate numerous shortage points $\left(O_{p s}\right) . O_{p s}$ represents the shortage point $s$ of part $p$. It is also the latest delivery time of part $p$. If the arrival time exceeds this time, parts shortage will occur.
5.2. Arrange Initial Tour. According to type of parts to be transported, the minimum number of tours $(N)$, and limit capacity of tow train, two decisions need to be solved in arranging initial tour. It is important to determine type of parts and start time for each tour.

First step: determine type of parts. Sort shortage point $\left(O_{p s}\right)$ in ascending order. Because the capacity limit of tow train is $C$, the type of parts should not exceed the capacity $C$ at each tour. Determine part type for each tour in turn.

Second step: determine the start time of each tour. According to the shortage point of each part $\left(O_{p s}\right)$, driving time from supermarket to part $p\left(t_{p}\right)$, and unloading time of part bins $(R)$, it is efficient to obtain latest start time $\left(S_{n p}\right)$ by using backward induction, $S_{n p}=O_{p s}-t_{p}-R$, and then, obtain start time of each tour, $t_{n}=\min \left\{S_{n p}\right\}$. Starting from this time, all parts will not be out of stock during the tour. And it can make the time for part to arrive at stations as close as possible to the time it consumes. Calculate the end time of tour, $E_{n}=t_{n}+R+T . R$ is the total time for unloading all part bins in one tour, $S_{n p}$ is the start time of tour $n$ based on part $p$, and $E_{n}$ is the end time of tour $n$.

5.3. Tow Train Allocation. After determining type of parts and start time of each tour, it is necessary to make decisions on the number of tow trains to be distributed. The indispensable step is to ensure that tow train completes all the tours through reasonable planning.

First step: calculate the number of tow trains $(K)$. The total production time $\left(t_{e}\right)$ can be obtained by the number of cycles $(M)$ and cycle time $(c t)$ of assembly planning period. Then, an initial number of tow trains can be calculated according to the following equation:

$$
K=\left\lceil N /\left(\frac{M * c t}{T+R+L}\right)\right\rceil .
$$

Second step: tow train transport allocation. Sort the start time $\left(t_{n}\right)$ in ascending order, so it could obtain an array of new start time. Split new start time array into same small arrays as the number of tow trains. Get a new start time $\left(t_{n k}\right)$ and end time $\left(E_{n k}\right)$ by tow train $k . E_{n k}$ is the end time when tow train $k$ performs tour $n$. For example, if the number of tow trains is 2, array of start time for sorting is divided into odd and even columns. Odd column is arranged for the first tow train to be transported, and even column is arranged for the second tow train. Through this allocation method, the interval between two tours performed by the same tow train is increased, and the possibility of shortage is reduced.

5.4. Transport Start Time Coordination. After tow train transport allocation, an overlapping tour may generate, and it is essential to coordinate overlapping tour start time.

First step: judge overlapping tour according to tow train. Overlapping refers to two tours performed by the same tow train. The current tour has not ended yet, but the next tour start time has been reached, $E_{n-1 k}>t_{n k}$. According to the result of the tow train allocation process, 


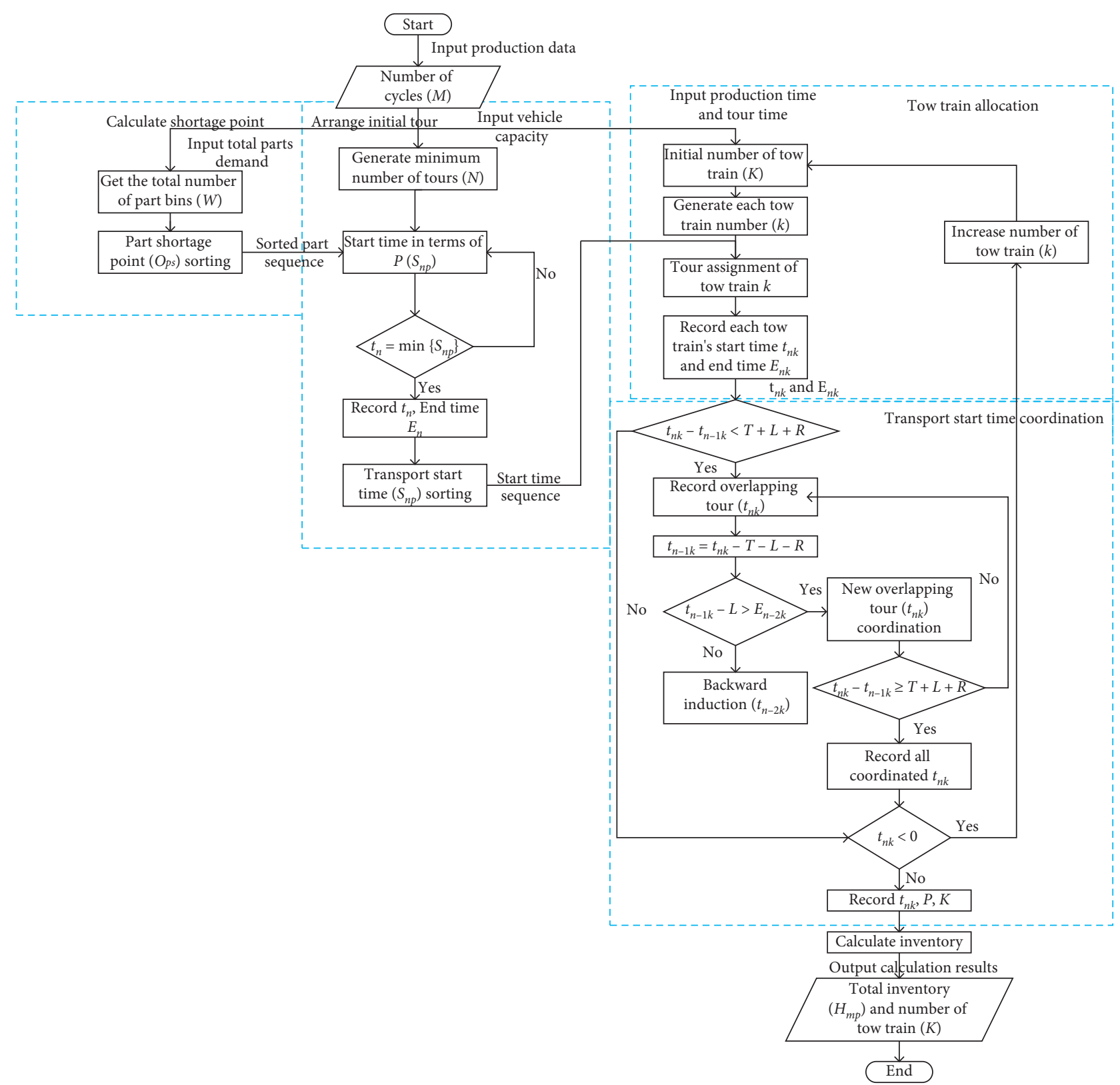

FIgURE 2: Flow chart of heuristic algorithm.

the start time and end time of tour $n$ performed by tow train $k$ can be obtained. Calculate the number of overlapping tours by tow train and output the serial number of overlapping tours.

Second step: transport start time coordination. Search the last two overlapping tours according to a tow train. It is required to ensure that the last start time does not change. Obtain start time of its previous tour by using a backward induction, $t_{n-1 k}=t_{n k}-T-L-R$. If the conditions are met $t_{n-}$ $1 k-L>E_{n-2 k}$, it will complete tour coordination. Otherwise, obtain start time of tour $t_{n-2 k}$ by using backward induction until the condition is met; then, coordination is completed. Next, find new overlapping tours for coordination until all overlapping tours are coordinated, $t_{n k}-t_{n-1 k}>=T+L+R$.
Update coordinated start time $t_{n k}$. $L$ is the total time for loading all part bins in one tour.

Third step: increase the number of tow trains. Output coordinated $t_{n k}$ in the second step; if $t_{n k}<0$, it indicates that start time is negative. Since the current number of tow trains is too small to complete all tours, it is essential to increase the number of tow trains. Then, perform tow train allocation process again until appropriate start time $\left(t_{n k}\right)$ is found. In this process, if the number of tow trains does not need to increase, that is, the initial number of tow trains is the smallest number of vehicles. When it is necessary to increase the number of tow trains, it means that the initial number of vehicles cannot complete all the tours, and the increased number of tow trains is the near optimal number of vehicles. 
TABLE 5: BOM of models.

\begin{tabular}{lcccccccccccccc}
\hline & $P_{1}$ & $P_{2}$ & $P_{3}$ & $P_{4}$ & $P_{5}$ & $P_{6}$ & $P_{7}$ & $P_{8}$ & $P_{9}$ & $P_{10}$ & $P_{11}$ & $P_{12}$ & $P_{13}$ & $P_{14}$ \\
\hline$M_{1}$ & 1 & 1 & 1 & 1 & 0 & 0 & 1 & 1 & 0 & 0 & 1 & 0 & 0 & 1 \\
$M_{2}$ & 1 & 0 & 0 & 0 & 1 & 1 & 1 & 0 & 1 & 0 & 0 & 1 & 0 & 1 \\
$M_{3}$ & 1 & 0 & 0 & 0 & 1 & 0 & 1 & 1 & 0 & 1 & 0 & 0 & 1 & 0 \\
$\mathrm{D} / \mathrm{m}$ & 163 & 172 & 184 & 300 & 303 & 406 & 479 & 582 & 600 & 710 & 730 & 745 & 848 & 920 \\
$Q_{p}$ & 18 & 4 & 5 & 5 & 6 & 6 & 41 & 22 & 5 & 19 & 42 & 42 & 12 & 44 \\
\hline
\end{tabular}

\section{Computational Study}

6.1. Instance Generation. Three different models $\left(M_{1}, M_{2}\right.$, and $M_{3}$ ) are assembled on a mixed model assembly line of a car manufacturer. There is a material supermarket next to assembly line. It has four tow trains that can service six stations $\left(S_{1} \sim S_{6}\right)$ along line. In this area, the assembly process requires fourteen different parts that can be supplied by tow trains. Each part at the station has an initial inventory. According to customer's order, assembly sequence is known in advance so that logistics worker can pick part bins. At the beginning of production, three models are successively assembled on stations according to model sequence. The cycle time is 72 seconds. Tow train loads part bins in supermarket, and the loading time for a bin is 37 seconds, waiting until appropriate time to carry out delivery task. It travels at a constant speed of $3 \mathrm{~m} / \mathrm{s}$ on a predefined route. When tow train passes through storage location of each part, it will unload corresponding part bins and then return to supermarket after completing delivery task; the unloading time for a bin is 43 seconds. Table 5 shows the bill of materials for three models, distance for tow train to drive from supermarket to part $p(D)$, and number of parts in a bin $\left(Q_{p}\right)$.

6.2. Computational Results. In order to tackle the mathematical model, we used a default solver, namely, CPLEX. And the maximum run time was set to 3600 seconds. Heuristic algorithm for just-in-time delivery is implemented in Java. All programs run on a computer configured as an Intel ${ }^{\circledR}$ Core $^{\mathrm{TM}}$ i7-8550U CPU processor and $8.00 \mathrm{~GB}$ of RAM. On the strength of changes in the number of production cycles, instances were generated with different scales. Small scales were 17 to 60 cycles, medium scales were 60 to 100 cycles, and when number of cycles exceeded 100, it would be a big-scale problem. When solving with CPLEX, firstly, we will get the minimum number of tow trains. Then, the minimum number of tow trains will be used as a parameter to further solve the mathematical model to obtain the minimum inventory. To evaluate computational performance of our proposed heuristic algorithm, it was pitted against CPLEX in small scales. Also, we compared the heuristic algorithm with genetic algorithm. During the assembly planning period, the number of parts to be delivered is coded as a chromosome by means of real number encoding. Decode chromosomes with parts sequences to obtain the number of tow trains and transportation scheme and calculate the inventory. The selection process uses a combination of elite retention strategies and roulette. The crossing process adopts single-point crossover. Through selection, crossover, and mutation, the optimal solution or approximate optimal solution is obtained after several iterations of optimization. In addition, results of just-in-time delivery are compared with periodic delivery. Periodic delivery is a fixed schedule of delivery vehicles on account of number of production cycles during the assembly planning period. The start time for tow train $k$ to perform tour $n$ is constant. The periodic delivery interval $t$ can be calculated according to the following formula:

$$
t=\left\lfloor(M * C) /\left\lceil\sum_{P=1}^{P}\left(D_{p}-h_{0 p}\right) / Q_{p}\right\rceil\right\rfloor .
$$

The calculation time and target value of the heuristic algorithm, genetic algorithm, and CPLEX at $C=3$ are shown in Table 6.

As it can be seen from Table 6, for the instance in which CPLEX can find optimal results in maximum run time, the heuristic algorithm also can obtain optimal or approximate optimal solutions in a short time, showing excellent performance. The optimal inventory solved by the heuristic algorithm is slightly larger than CPLEX only for the problem with 36 cycles and five tours. When objective function is set to minimize the number of tow trains, the computational complexity of the problem is reduced. Consequently, CPLEX can get a minimum number of tow trains for more instances during the maximum run time, but it failed to optimize corresponding inventory. In comparison with solution time of two modes of just-in-time delivery, the solution time of the heuristic algorithm is less than four seconds, and a better solution can be obtained to the problem in a short time compared with CPLEX. As the number of cycles increases, the advantage of the heuristic algorithm in solution time is more obvious. When CPLEX solves the minimum number of tow trains with 72 cycles, solution time has reached 1504.7 seconds and CPLEX cannot solve the optimal inventory in maximum run time. It could use "-_" to indicate that. However, the heuristic algorithm only takes 0.71 seconds to solve the optimal inventory while obtaining the minimum number of tow trains.

And the genetic algorithm also shows good performance in small-scale problems. Comparing these two algorithms, it is found that the heuristic algorithm is superior to the genetic algorithm in both solving speed and solution quality in almost all the instances except for 72 cycles and thirteen tours. With expansion of the problem scale, although the genetic algorithm can obtain smaller inventory, it has caused an increase in the number of vehicles. And the minimum number of tow trains is the primary objective, and the 


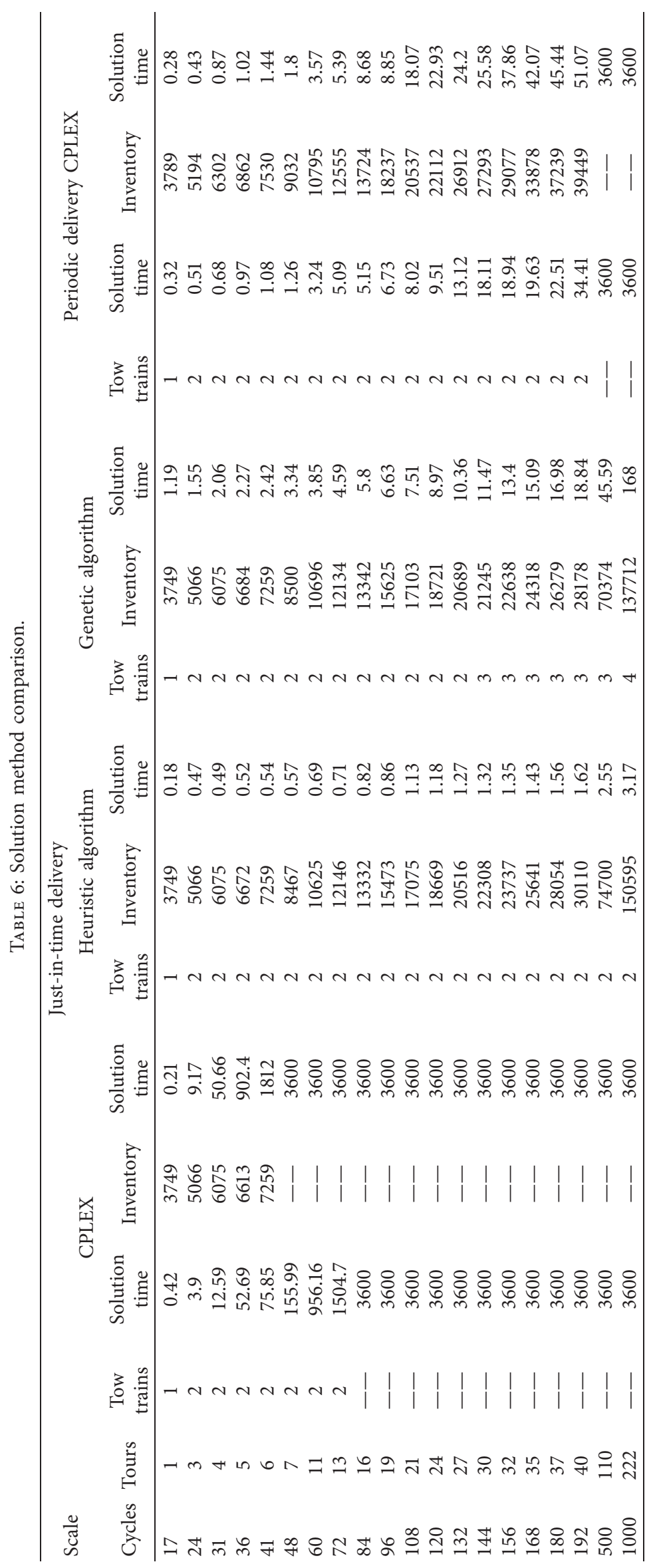




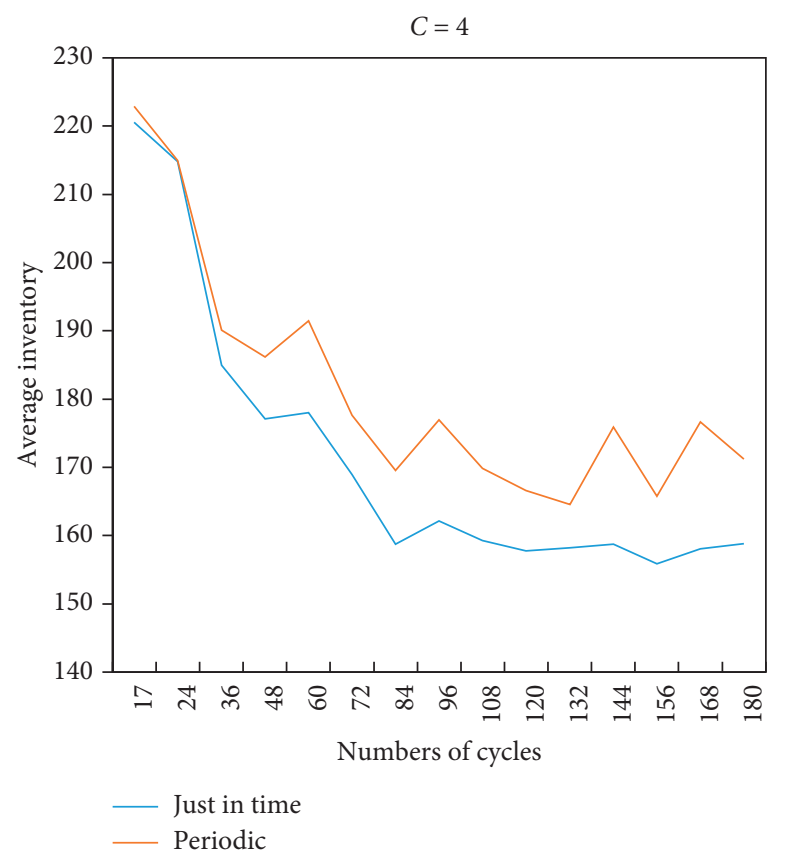

(a)

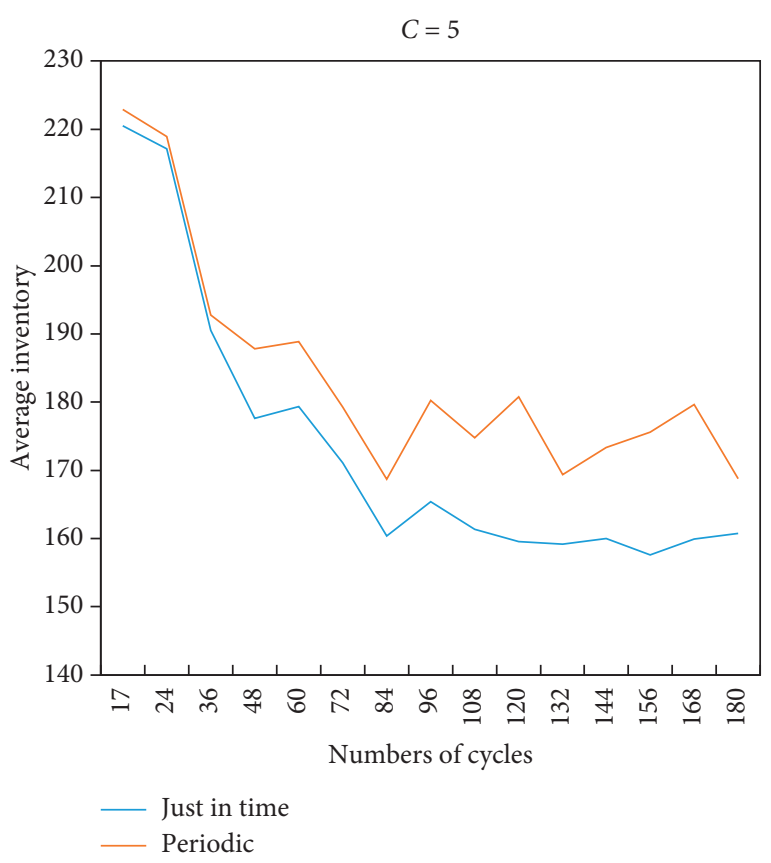

(b)

FIGURE 3: Average inventory of delivery strategies under different capacities of tow trains.

increase in the number of vehicles will cause a substantial increase in material distribution costs.

Making a comparison between two delivery methods, each tour starts at the same interval in periodic delivery, which simplifies decision on delivery time, and reduces computational complexity, so CPLEX can solve largerscale instances. Due to calculation results, both delivery methods can find the same minimum number of tow trains. However, just-in-time delivery can reasonably arrange tow train while obtaining the smallest tow train. It can reduce line-side inventory and makes inventory results significantly better than periodic delivery strategies.

6.3. Result Analysis. The average inventory level can be calculated by averaging the inventory level under each cycle. When capacity of tow train and number of tours are changed, it could analyze the impact of different delivery strategies on average inventory. Therefore, we set the tow train capacity to 4 and 5 and calculate average inventory; the line diagram is shown in Figure 3.

From Figure 3, when capacity of tow train is certain, as the number of cycles increases, average inventory of two delivery strategies shows a decreasing trend. Since initial inventory is set to one bin of each part, production can be maintained for a period of time, and initial inventory is higher than average inventory. For this reason, average inventory decreases significantly when increasing the number of cycles in the early stage and then appears as a relatively stable state. The average inventory performances of two delivery strategies under capacities $\mathrm{C}=4$ and 5 are similar to the results obtained by solving the problem at $C=3$. Average inventory of just-in-time delivery is significantly less than that of periodic delivery, which has more advantages in reducing inventory. In periodic delivery, start time is fixed, and flexibility is poor. It causes a large number of parts to be transported to stations in advance, resulting in an increased inventory.

The impact of capacity on number of tow trains and average inventory in just-in-time delivery strategy was analyzed. Thus, setting $C=3,4$, and 5 , the result of required number of tow trains and average inventory is shown in Figures 4 and 5 .

As the number of cycles increases, the number of tours increases. It can be seen from Figure 4, the number of tow trains for just-in-time delivery strategy under different capacities is increasing. However, tow train with capacity of 5 would require fewer than capacity of 3 or 4 in small-scale instance. At the same number of cycles, the smaller the capacity of tow train, the more the number of tours. Hence, more frequent transport is required. In Figure 5, as capacity of tow train increases, average inventory shows an increasing trend. It is obvious to find that using a tow train with a capacity of 3 can achieve a lower inventory in relation to a capacity of 4 and 5, which is in accordance with the tendency of using small batch to transport material. It can keep inventory at a lower level. However, when the number of cycles is small in early stage, more tow trains are needed when capacity is 3 or 4 . Consequently, according to number of cycles, appropriate capacity should choose to complete tours, which could reduce production costs by getting minimum inventory while using minimum number of tow trains. 


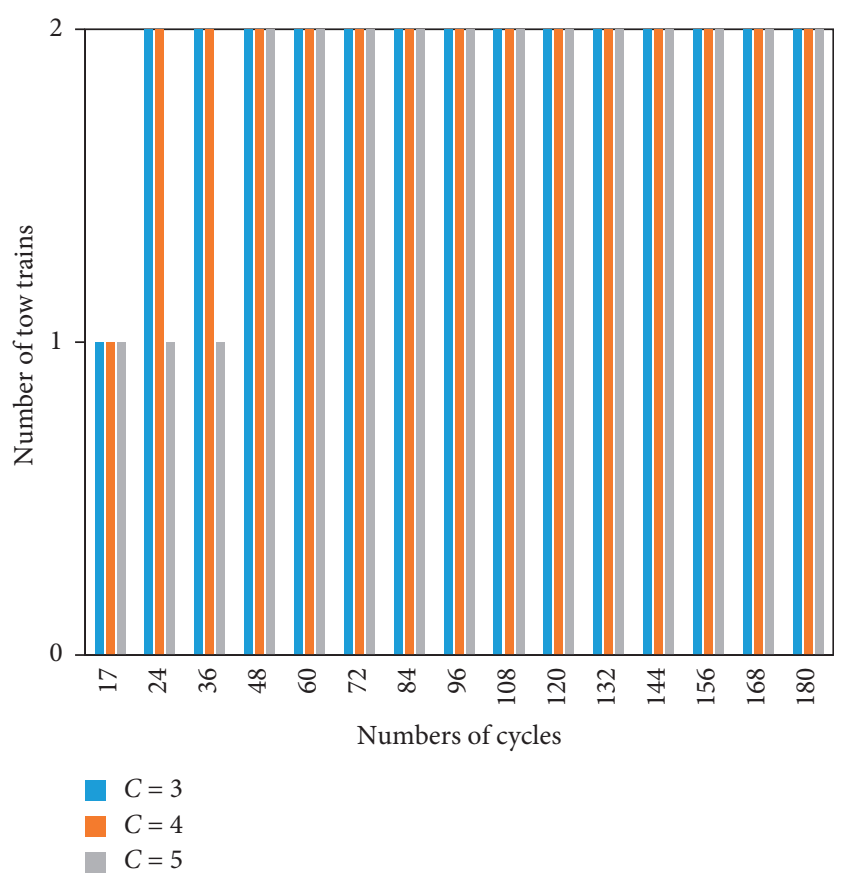

FIGURE 4: Number of vehicles in different capacities.

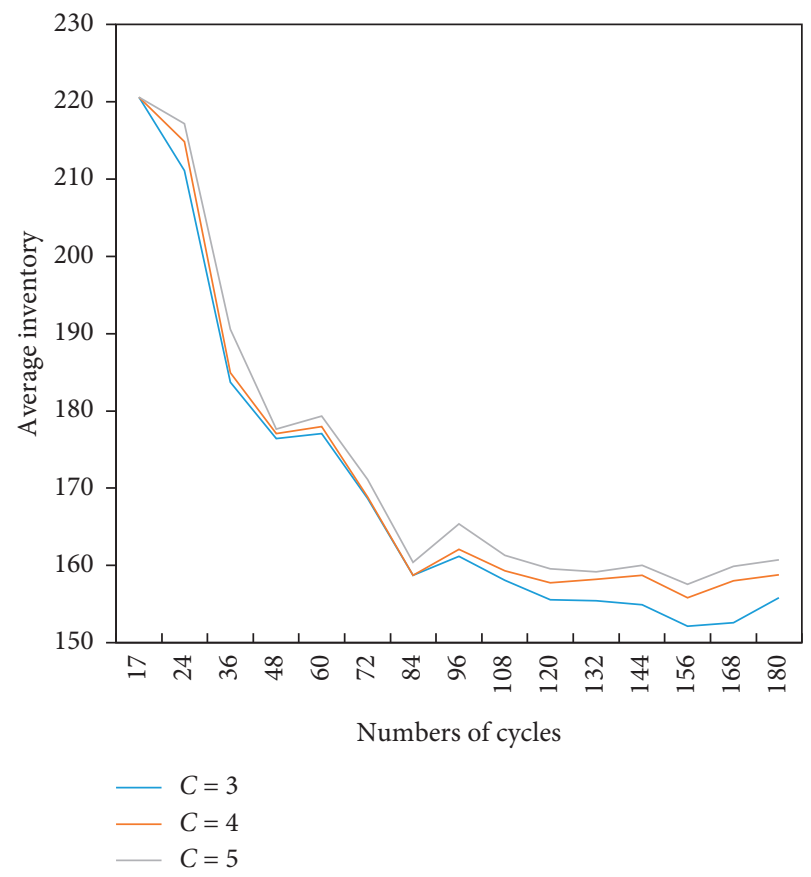

FIgURE 5: Average inventory in different capacities.

The analysis of results shows that parts are stored on the line side for the shortest time by using the just-in-time delivery strategy. It could ensure that tow train completes all tours without shortage. In contrast to traditional periodic delivery, it can effectively reduce inventory at stations. Furthermore, capacity of tow train has a greater impact on the number of tow trains and inventory levels. It is indispensable to select reasonable capacity according to number of cycles, thereby reducing costs of parts delivery.

\section{Conclusions and Prospects}

This study mainly studies the problem of tow train scheduling to feed parts for mixed model assembly line. Based on inventory of station and capacity of tow train, decisions of number of tow trains, schedule of each tours, and type of parts loaded per tour need to be made. Firstly, a just-in-time delivery model is established with the primary goal of minimizing the number of tow trains. Then, in view of the minimum number of tow trains, schedule of each tours is reasonably planned so that time at which parts arrive station is as close as possible to consumption time, and inventory level is lowered. As a result, a heuristic algorithm for problems is designed for large-scale instances. In contrast with traditional periodic delivery strategy, the results show that just-in-time delivery has more advantages in reducing inventory level. In just-in-time delivery strategy, different capacity of tow train has a greater impact on inventory level, and tow trains with small capacity can obtain lower inventory levels, which is consistent with using multifrequency and small-batch methods to transport materials.

At present, the concept of just-in-time delivery with a material supermarket is applied in automobile manufacturing. Although the just-in-time delivery model established in this paper is very complicated, it also simplifies the actual material scheduling problem. In actual assembly production, the tow train breakdown and logistics space congestion will affect the distribution process. At the same time, the vehicle driving speed, loading, and unloading time also fluctuate. In future research, we can consider further improving the mathematical model from vehicle breakdown and logistics space congestion. And we could also make improvement in algorithm in order to adapt a more flexible delivery.

\section{Data Availability}

The data used to support the findings of this study are included within the article.

\section{Conflicts of Interest}

The authors declare that they have no conflicts of interest.

\section{Acknowledgments}

This work was supported by the National Natural Science Foundation of China (Grants nos. 51405283, 71401098, and 71572104) and the China Scholarship Council (no. 201906895026).

\section{References}

[1] D. Battini, N. Boysen, and S. Emde, "Just-in-time supermarkets for part supply in the automobile industry," Journal of Management Control, vol. 24, no. 2, pp. 209-217, 2013.

[2] H. Y. K. Lau and S. O. Woo, "An agent-based dynamic routing strategy for automated material handling systems," International Journal of Computer Integrated Manufacturing, vol. 21, no. 3, pp. 269-288, 2008. 
[3] H. S. Kilic and M. B. Durmusoglu, "Advances in assembly line parts feeding policies: a literature review," Assembly Automation, vol. 35, no. 1, pp. 57-68, 2015.

[4] N. Boysen, S. Emde, M. Hoeck, and M. Kauderer, "Part logistics in the automotive industry: decision problems, literature review and research agenda," European Journal of Operational Research, vol. 242, no. 1, pp. 107-120, 2015.

[5] D. Battini, M. Gamberi, A. Persona, and F. Sgarbossa, "Partfeeding with supermarket in assembly systems: transportation mode selection model and multi-scenario analysis," Assembly Automation, vol. 35, no. 1, pp. 149-159, 2015.

[6] D. Battini, M. Faccio, A. Persona, and F. Sgarbossa, "Supermarket warehouses: stocking policies optimization in an assembly-to-order environment," The International Journal of Advanced Manufacturing Technology, vol. 50, no. 5-8, pp. 775-788, 2010.

[7] S. Emde and N. Boysen, "Optimally locating in-house logistics areas to facilitate JIT-supply of mixed-model assembly lines," International Journal of Production Economics, vol. 135, no. 1, pp. 393-402, 2012.

[8] S. Emde, M. Fliedner, and N. Boysen, "Optimally loading tow trains for just-in-time supply of mixed-model assembly lines," IIE Transactions, vol. 44, no. 2, pp. 121-135, 2012.

[9] B. S. Vaidyanathan, J. O. Matson, D. M. Miller, and J. E. Matson, "A capacitated vehicle routing problem for justin-time delivery," IIE Transactions, vol. 31, no. 11, pp. 1083-1092, 1999.

[10] W. Choi and Y. Lee, "A dynamic part-feeding system for an automotive assembly line," Computers \& Industrial Engineering, vol. 43, no. 1-2, pp. 123-134, 2002.

[11] S. Emde, "Optimally routing and scheduling tow trains for JIT-supply of mixed-model assembly lines," European Journal of Operational Research, vol. 217, no. 2, pp. 287-299, 2012.

[12] Y.-Q. Rao, M.-C. Wang, K.-P. Wang, and T.-M. Wu, "Scheduling a single vehicle in the just-in-time part supply for a mixed-model assembly line," Computers \& Operations Research, vol. 40, no. 11, pp. 2599-2610, 2013.

[13] M. Fathi, A. Syberfeldt, M. Ghobakhloo, and H. Eskandari, "An optimization model for material supply scheduling at mixed-model assembly lines," Procedia CIRP, vol. 72, pp. 1258-1263, 2018.

[14] J. Golz, R. Gujjula, H.-O. Günther, S. Rinderer, and M. Ziegler, "Part feeding at high-variant mixed-model assembly lines," Flexible Services and Manufacturing Journal, vol. 24, no. 2, pp. 119-141, 2012.

[15] J. Müller, "Approximative solutions to the bicriterion vehicle routing problem with time windows," European Journal of Operational Research, vol. 202, no. 1, pp. 223-231, 2010.

[16] Q. Zhong, R. M. Lusby, J. Larsen, Y. Zhang, and Q. Peng, "Rolling stock scheduling with maintenance requirements at the Chinese High-Speed Railway," Transportation Research Part B: Methodological, vol. 126, pp. 24-44, 2019.

[17] S. Emde and M. Schneider, "Just-in-time vehicle routing for in-house part feeding to assembly lines," Transportation Science, vol. 52, no. 3, 2018.

[18] Y. Zhang, Q. Peng, Y. Yao, X. Zhang, and X. Zhou, "Solving cyclic train timetabling problem through model reformulation: extended time-space network construct and alternating direction method of Multipliers methods," Transportation Research Part B: Methodological, vol. 128, pp. 344-379, 2019.

[19] H. S. KilicM. B. Durmusoglu et al., "A mathematical model and a heuristic approach for periodic material delivery in lean production environment," The International Journal of
Advanced Manufacturing Technology, vol. 69, no. 5-8, pp. 977-992, 2013.

[20] M. Fathi, V. Rodríguez, D. B. M. M. Fontes et al., "A modified particle swarm optimisation algorithm to solve the part feeding problem at assembly lines," International Journal of Production Research, vol. 54, no. 3, pp. 1-16, 2017.

[21] S. Emde and M. Gendreau, "Scheduling in-house transport vehicles to feed parts to automotive assembly lines," European Journal of Operational Research, vol. 260, no. 1, pp. 255-267, 2017.

[22] M. Fathi, M. J. Alvarez, F. Hassani Mehraban et al., "A multiobjective optimization algorithm to solve the part feeding problem in mixed-model assembly lines," Mathematical Problems in Engineering, vol. 2014, Article ID 654053, 12 pages, 2014.

[23] N. Boysen and S. Emde, "Scheduling the part supply of mixedmodel assembly lines in line-integrated supermarkets," European Journal of Operational Research, vol. 239, no. 3, pp. 820-829, 2014.

[24] R. Accorsi, R. Manzini, and F. Maranesi, "A decision-support system for the design and management of warehousing systems," Computers in Industry, vol. 65, no. 1, pp. 175-186, 2014.

[25] B.-H. Zhou and C.-Y. Shen, "Multi-objective optimization of material delivery for mixed model assembly lines with energy consideration," Journal of Cleaner Production, vol. 192, pp. 293-305, 2018.

[26] L. Qiu, J. Wang, W. Chen et al., "Heterogeneous AGV routing problem considering energy consumption," in Proceedings of the 2015 IEEE International Conference on Robotics and Biomimetics (ROBIO), IEEE, Zhuhai, China, pp. 1894-1899, December 2015. 